\title{
Association of Dietary Intake Ratio of n-3/n-6 Polyunsaturated Fatty Acids with Breast Cancer Risk in Western and Asian Countries: A Meta-Analysis
}

\author{
Ricvan Dana Nindrea ${ }^{1,2 *}$, Teguh Aryandono ${ }^{3}$, Lutfan Lazuardi ${ }^{4}$, Iwan \\ Dwiprahasto 5
}

\begin{abstract}
Objective: This systematic review and meta-analysis were performed to determine association of dietary intake ratio of n-3/n-6 polyunsaturated fatty acids with breast cancer risk in Western and Asian countries. Methods: The authors conducted a meta-analysis of published research articles on association of dietary intake ratio of $n-3 / n-6$ polyunsaturated fatty acids (PUFAs) with breast cancer risk in Western and Asian countries published between January 2000 and February 2019 in online article databases of PubMed, ProQuest and EBSCO. Pooled risk ratios (RR) were calculated using fixed and random-effect models. Publication bias was visually evaluated by performing funnel plots and statistically assessed by Egger's and Begg's tests. Data were processed by using Stata version 14.2 (Stata Corporation). Results: This study reviewed 913 articles. There were 13 studies included in systematic review continued by meta-analysis of relevant data with total number of samples: 275,264 patients. The results showed dietary intake ratio of n-3/n-6 PUFAs with breast cancer risk in Western and Asian countries $(\mathrm{RR}=0.99 ; 95 \%$ CI: 0.92-1.07). Dietary intake ratio of $n-3 / n-6$ PUFAs with breast cancer risk in Western countries reached (RR $=0.98 ; 95 \%$ CI: $0.91-1.06)$ and there was any significant publication bias for studies included. Dietary intake ratio of $n-3 / n-6$ PUFAs with breast cancer risk in Asian countries reached ( $\mathrm{RR}=1.18 ; 95 \% \mathrm{CI}$ : $0.94-1.47)$ and there was not any significant publication bias for studies included. Conclusion: This analysis confirmed association of dietary intake ratio of n-3/n-6 PUFAs with breast cancer risk in Western and Asian countries. Higher dietary intake ratio is associated with lower risk of breast cancer in Asian countries rather than Western countries. This study suggests increasing dietary intake ratio n-3/n-6 PUFAs will provide benefit for breast cancer prevention.
\end{abstract}

Keywords: Breast cancer- omega-3- omega-6- polyunsaturated fatty acids

Asian Pac J Cancer Prev, 20 (5), 1321-1327

\section{Introduction}

Breast cancer has become a major health problem in developing and developed countries. The increase of breast cancer incidence in western countries is higher compared to low-risk ones such as Japan, Singapore and urban areas in China. Lately, almost double increase was found in Asian countries incidence due to the lifestyle change and rapid economic growth (Willett, 2000; Nindrea et al., 2017; Nindrea et al., 2019).

Breast cancer is known to have multifactorial causes. The role of dietary factors in the risk of breast cancer has been the focus in several epidemiological studies. Breast cancer incidence in Asian women is lower than Western women due to high consumption of fish oil. This study shows that environmental factors and changes in eating habits contribute to the rise of breast cancer risk. These findings provide preliminary evidence that environmental factors, such as diet, may change the disease risk factors (MacLennan and Ma, 2010; Nindrea et al., 2018; Nindrea et al., 2019).

The components of dietary that related to the occurrence of breast cancer are fatty acids which provide a direct effect. Unlike proteins and carbohydrates, fatty acids are incorporated directly into the membrane. Some types of essential fatty acids (omega-3 and omega-6) cannot be synthesized by mammals. Dietary is essential as a risk factor for breast cancer. Various fatty acids, both saturated and unsaturated, are all potentially related to increased cancer risk. Polyunsaturated fatty acids (PUFAs) consist of two classes: omega- 6 (n-6 PUFAs) and omega-3 (n-3 PUFAs). Linoleic acid (LA, 18: 2n6) and arachidonic 
acid (AA, 20: 4n6) are two omega-6 PUFAs contained in the diet and may trigger cancer (Yang et al., 2014; Zheng et al., 2013).

The high amount of omega-6 PUFA and the very high ratio of omega- 6 / omega- 3 as in the western diet, has been shown to trigger the pathogenesis of various cancers including breast cancer, whilst increased omega-3 PUFA levels (omega-6 ratio / low omega-3) has been widely known for producing suppressive effects. However, there is still a debate to consider whether the decreasing of omega-6 PUFA diet or the lower ratio of omega-6 / omega-3 would give a better effect (Liu and Ma., 2014).

A study by Yang et al., (2014) found that higher intake ratio of n-3/n-6 PUFAs is associated with lower risk of breast cancer. However, some subsequent studies of n-3/n-6 PUFAs and breast cancer led to contradictory conclusions (Murff et al., 2011; Engeset et al., 2006). As a result, the current research findings seem to be inconsistent. The different results possibly happened due to different dietary patterns of $n-3 / n-6$ ratio polyunsaturated fatty acids in Asian and Western populations, for whom the latter has lower intake, which may impact the significance of the results.

Therefore, we aimed to determine association of dietary intake ratio of n-3/n- 6 polyunsaturated fatty acids with breast cancer risk in Western and Asian countries by conducting a meta-analysis study in order to draw stronger conclusions.

\section{Materials and Methods}

\section{Study design and research sample}

This research was a quantitative research with meta-analysis study design. The meta-analysis followed the preferred reporting items from the Systematic Reviews and Meta-Analysis (PRISMA) guideline (Liberati et al., 2009). Meta-analysis was used to figure association of dietary intake ratio of n-3/n-6 polyunsaturated fatty acids with breast cancer risk in Western and Asian countries. The research samples were published research articles published between January 2000 and February 2019 in online article databases of PubMed, ProQuest and EBSCO.

\section{Operational definitions}

The variables of this study included an independent variable which was n-3/n-6 polyunsaturated fatty acids and a dependent variable which was breast cancer risk.

\section{Research procedure}

This study was conducted by collecting data through the identification of published research articles on association of dietary intake ratio of n-3/n- 6 polyunsaturated fatty acids with breast cancer risk in Western and Asian countries based on online article databases of PubMed, ProQuest and EBSCO (Figure 1). We used the following key words treated as title/abstract for the literature search: ("fat" OR "fatty acid" OR "alpha-linolenic acid" OR "polyunsaturated fatty acid" OR "omega-3 fatty acid" OR "n-3 fatty acid" OR "omega-6 fatty acid" OR "n-6 fatty acid" OR "ratio") AND ("breast cancer" OR "breast neoplasms").

Identification of 913 articles was done by reviewing through the titles of the articles, continued by reviewing the abstracts, and then the full text form. The article was excluded if: (a) not relevant subject outcome, (b) not case control and cohort study, and/or (c) the information provided in the results was not enough or insufficient for data extraction.

\section{Data collection technique}

The data collection was done through online search. The search was limited to English language articles. The article type was limited to journal article. The research subject was limited to research with human subject. The time of publication was limited from January 2000 to February 2019. The abstract of articles with potentially relevant titles were reviewed, while the irrelevant articles were excluded. Furthermore, articles which have potentially relevant abstracts then reviewed in full-text, whilst the irrelevant articles were excluded. The inclusion criteria of this study sample were research on association of dietary intake ratio of n-3/n- 6 polyunsaturated fatty acids with breast cancer risk in Western and Asian countries with case control and cohort study methods. Exclusion criteria were: the research was not available in full text form and if inclusion criteria were unfulfilled or if the information were insufficient for data extraction. The following data were obtained from each article: first author's name and year of publication, region, type of study, number of samples, pre or postmenopause and effect size.

Two independent investigators carefully extracted information from all studies that fulfilled the inclusion criteria in accordance with a standardized protocol. Disagreements were resolved by three other investigators. Quality assessment was conducted using Newcastle-Ottawa Quality Assessment Scale (NOS). The papers with a total score of 0-3, 4-6, and 7-9 points were specified as the poor, moderate, and high quality (Wells et al., 2009).

\section{Data analysis}

The analysis was held to get the value of pooled risk ratio which is the combination of risk ratio value from each research. Results were pooled risk ratio with corresponding 95\% confidence intervals (CIs). Significant heterogeneity was indicated by $\mathrm{I}^{2}>50 \%$ because these tests had minimal statistical power in cases with few studies and small sample sizes. A random effect model was used when significant heterogeneity was observed; otherwise, a fixed effect model was performed. Publication bias was visually evaluated by using funnel plots and statistically assessed through Egger's and Begg's tests. Meta-analysis used Stata version 14.2 (Stata Corporation). A two-tailed p-value of $<0.05$ was considered statistically significant.

\section{Results}

The identification of the published articles was conducted and we obtained 13 studies with number of 
samples 275,264 patients related to dietary intake ratio of n-3/n- 6 polyunsaturated fatty acids with breast cancer risk in Western and Asian countries (Table 1).

Figure 2 shows the meta-analysis of articles on dietary intake ratio of n-3/n- 6 polyunsaturated fatty acids with breast cancer risk in Western and Asian countries $(\mathrm{RR}=0.99$ [95\% CI 0.92-1.07]). Heterogeneity test for studies on dietary intake ratio of $n-3 / n-6$ polyunsaturated fatty acids with breast cancer risk in Western and Asian countries (Pheterogeneity $=0.024 ; \mathrm{I} 2=48.8 \%$ ) showed variation of homogeneous research. Funnel plots for identify publication bias among studies dietary intake ratio of n-3/n- 6 polyunsaturated fatty acids with breast cancer risk in Western and Asian countries was held (Figure 3).

Figure 3 described that there was not any significant publication bias for studies included in articles related to dietary intake ratio of n-3/n- 6 polyunsaturated fatty acids with breast cancer risk in Western and Asian countries, Egger's test ( $p=0.051)$ and Begg's test $(p=0.067)$.

Table 2 shows association of dietary intake ratio of n-3/n-6 polyunsaturated fatty acids with breast cancer risk in Western and Asian countries $(\mathrm{RR}=0.98[95 \%$ CI 0.91-1.06]). Heterogeneity among studies on dietary intake ratio of $n-3 / n-6$ polyunsaturated fatty acids and breast cancer risk in Western countries (Pheterogeneity
$=0.013 ; \mathrm{I} 2=57.0 \%$ ) showed variation of heterogeneous research. There was significant publication bias for studies included in dietary intake ratio of $n-3 / n-6$ polyunsaturated fatty acids with breast cancer risk between in Western countries, Egger's test ( $\mathrm{p}=<0.001)$ and Begg's test ( $\mathrm{p}$ $=0.009$ ). We also found dietary intake ratio of $n-3 / n-6$ polyunsaturated fatty acids with breast cancer risk in Asian countries ( $\mathrm{RR}=1.18$ [95\% CI 0.94-1.47]). Heterogeneity among studies on dietary intake ratio of n-3/n-6 polyunsaturated fatty acids with breast cancer risk in Asian countries (Pheterogeneity $=0.117 ; \mathrm{I} 2=47.2 \%$ ) showed variation of homogeneous research. There was not any significant publication bias for studies included in dietary intake ratio of n-3/n-6 polyunsaturated fatty acids with breast cancer risk in Asian countries, Egger's test $(p=0.240)$ and Begg's test $(p=0.117)$.

\section{Discussion}

This analysis confirmed association of dietary intake ratio of n-3/n-6 PUFAs with breast cancer risk in Western and Asian countries ( $\mathrm{RR}=0.99$ [95\% CI 0.92-1.07]). Higher dietary intake ratio is associated with lower risk of breast cancer in Asian countries rather than Western countries.

Table 1. Systematic Review on Dietary Intake Ratio of n-3/n-6 Polyunsaturated Fatty Acids and Breast Cancer Risk in Western and Asian Countries

\begin{tabular}{|c|c|c|c|c|c|c|c|c|}
\hline \multirow[t]{2}{*}{ First author, year } & \multirow[t]{2}{*}{ Region } & \multirow{2}{*}{$\begin{array}{l}\text { Type of } \\
\text { study }\end{array}$} & \multicolumn{2}{|c|}{ Number of samples } & \multirow{2}{*}{$\begin{array}{l}\text { Pre-or-Post } \\
\text { menopause }\end{array}$} & \multirow[t]{2}{*}{ Measurement } & \multirow[t]{2}{*}{ Effect size } & \multirow[t]{2}{*}{ NOS } \\
\hline & & & Cases & Controls & & & & \\
\hline $\begin{array}{l}\text { Saadatian-Elahi et } \\
\text { al., } 2002\end{array}$ & USA & $\mathrm{NCC}$ & 197 & 197 & Both & $\begin{array}{l}\text { n-3/n-6:Serum } \\
\text { PL,GC(\% tFC })\end{array}$ & RR (95\% CI):0.42 (0.17-1.08) & 7 \\
\hline Wirfalt et al., 2002 & Sweden & $\mathrm{NCC}$ & 237 & 673 & Post & $\begin{array}{c}\text { n-3/n-6:Diet, FFQ } \\
\text { (g/day) }\end{array}$ & RR (95\% CI):0.66 (0.41-1.08) & 7 \\
\hline Bagga et al., 2002 & USA & $\mathrm{CC}$ & 73 & 74 & Both & $\begin{array}{c}\text { n-3/n-6:Diet, } \\
\text { serum PL }(\% \text { tFC })\end{array}$ & OR $(95 \%$ CI):0.59 (0.32-1.10) & 6 \\
\hline $\begin{array}{l}\text { Goodstine et al., } \\
2003\end{array}$ & USA & $\mathrm{CC}$ & 565 & 554 & Both & $\begin{array}{c}\text { n-3/n-6:Diet, FFQ } \\
\text { (g/day) }\end{array}$ & OR $(95 \% \mathrm{CI}): 0.50(0.27-0.95)$ & 7 \\
\hline $\begin{array}{l}\text { Gago-Dominguez et } \\
\text { al., } 2003\end{array}$ & Singapore & $\mathrm{PC}$ & 314 & 314 & Both & $\begin{array}{c}\text { n-6/n-3: Diet, FFQ } \\
\text { (g/day) }\end{array}$ & RR $(95 \%$ CI):1.37 (1.06-3.27) & 7 \\
\hline Wakai et al., 2005 & Japan & $\mathrm{PC}$ & 13,145 & 13,146 & Both & $\begin{array}{l}\text { n-6/n-3: Diet, FFQ } \\
\text { ( } \% \text { energy) }\end{array}$ & RR $(95 \%$ CI):1.31 (0.78-2.19) & 8 \\
\hline Chajes et al., 2008 & Sweden & $\mathrm{NCC}$ & 363 & 702 & Both & $\begin{array}{l}\text { n-6/n-3:Serum } \\
\text { PL,GC (\% tFC) }\end{array}$ & RR (95\% CI):0.76 (0.48-1.20) & 7 \\
\hline Thiebaut et al., 2009 & France & $\mathrm{PC}$ & 56,007 & - & Both & $\begin{array}{c}\text { n-6/n-3:Diet, } \\
\text { FFQ,(\%energy) }\end{array}$ & RR (95\% CI):0.97 (0.83-1.14) & 7 \\
\hline Takata et al., 2009 & USA & $\mathrm{NCC}$ & 130 & 257 & Post & $\begin{array}{l}\text { n-3/n-6:Serum } \\
\text { PL,GC }(\% \text { tFC })\end{array}$ & RR (95\% CI):0.74 (0.40-1.36) & 8 \\
\hline Murff et al., 2011 & China & $\mathrm{PC}$ & 712 & 71,859 & Post & $\begin{array}{c}\text { n-6/n-3: Diet, FFQ } \\
\text { (g/day) }\end{array}$ & RR (95\% CI):1.02 (0.77-1.34) & 8 \\
\hline $\begin{array}{l}\text { Sczaniecka et al., } \\
2012\end{array}$ & USA & $\mathrm{PC}$ & 772 & 29,480 & Post & $\begin{array}{l}\text { n-3/n-6:Diet, } \\
\text { FFQ,(g/day) }\end{array}$ & RR (95\% CI):0.84 (0.65-1.09) & 8 \\
\hline Park et al., 2012 & USA & $\mathrm{PC}$ & 85,089 & - & Post & $\begin{array}{l}\text { n-6/n-3:Diet, FFQ, } \\
\quad(\mathrm{g} / 1000 \mathrm{kcal})\end{array}$ & RR $(95 \%$ CI):1.10 (0.99-1.22) & 8 \\
\hline Pouchieu et al., 2014 & France & $\mathrm{NCC}$ & 154 & 250 & Both & $\begin{array}{c}\mathrm{n}-3 / \mathrm{n}-6: \text { PLA, } \\
(\% \mathrm{tFC})\end{array}$ & RR (95\% CI):0.91 (0.52-1.58) & 7 \\
\hline Number of samples & & & 157,758 & 117,506 & & & & \\
\hline
\end{tabular}

$\%$ otFC, percentage of total Fatty Acid; GC, Gas Chromatography; CC, case-control study; NCC, prospective nested case-control study; $P C$, prospective cohort study; $n-3 / n-6$, ratio of $n-3 / n$ 6 polyunsaturated fatty acids; $n-6 / n-3$, ratio of $n-6 / n-3$ polyunsaturated fatty acids; FFQ, food frequency questionnaire; Serum PL, serum phospholipids; PLA, plasma fatty acids; NOS, NewcastleOttawa Quality Assessment Scale; OR, odds ratio; RR, Relative Risk; CI, Confidence Interval 


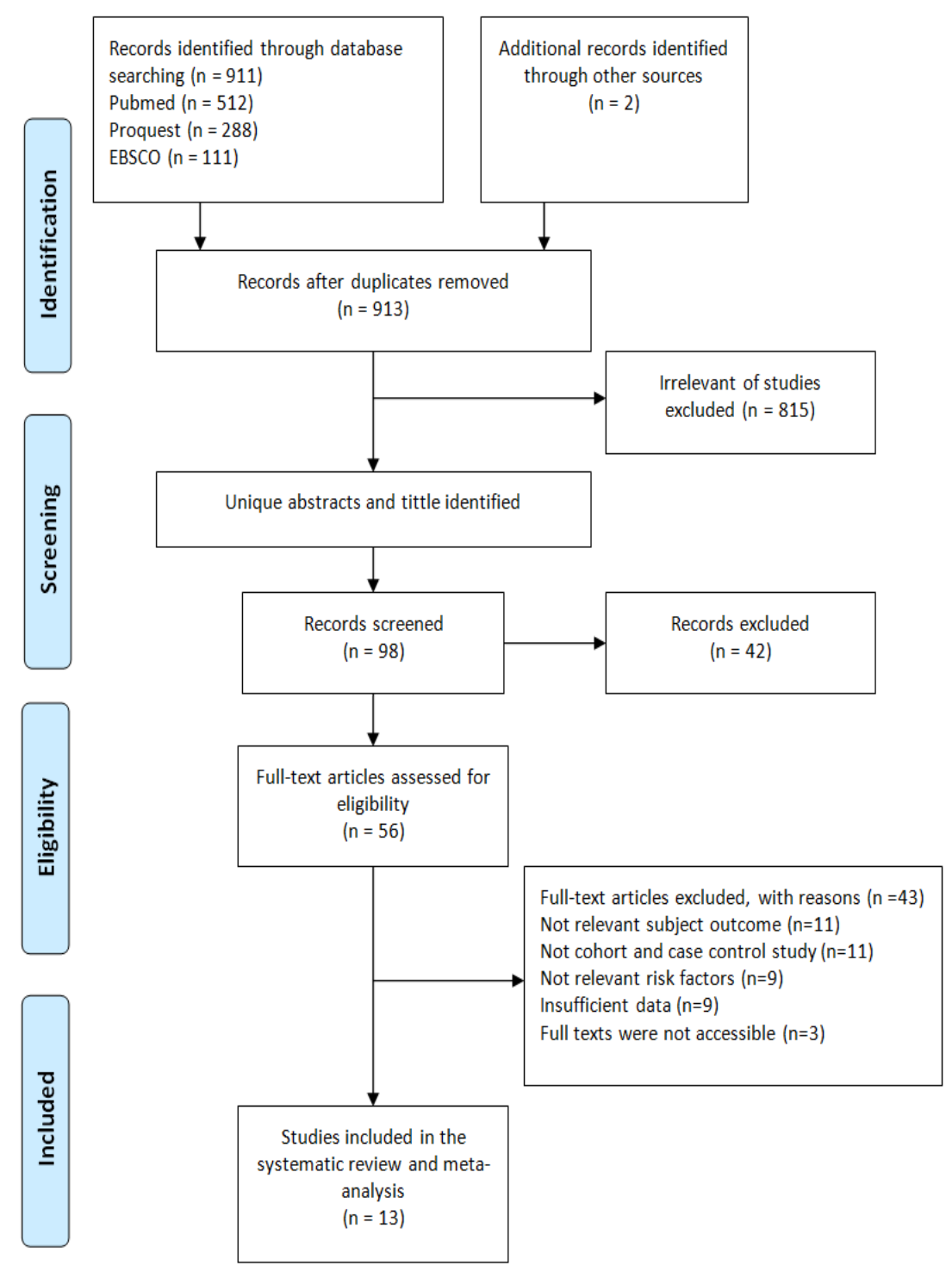

Figure 1. Flow Diagram Research Procedure

Previous study with meta-analysis of 26 international studies involving 883,585 female participants, including 20,000 breast cancer patients, found that omega- 3 fatty acids intake from fish provided $14 \%$ reduction of breast cancer risk (Zheng et al., 2013). Another study by Yang et al., (2014), has found that by increasing the ratio of omega-3 and omega 6 intake, lower risk of breast cancer could be obtained. The study also suggested higher ratio of omega-3 and omega 6 intake as deterrent factor of breast cancer.

Previous studies found that higher intake ratio of n-3/n-6 PUFAs is associated with lower risk of breast cancer (Chajes et al., 2008; Thiebaut et al., 2009; Takata et al., 2009; Sczaniecka et al., 2012; Pouchieu et al., 2014).
However, some subsequent studies of n-3/n-6 PUFAs and breast cancer led to contradictory conclusions (Murff et al., 2011; Engeset et al., 2006). It has been known that dietary intake n3-/n6 PUFAs of Asian population is considerably higher than Western populations (Terry et al., 2003; Fabian et al., 2015). Therefore, dietary intake in Western populations might not give significant effect on breast cancer risk as well as prove to be unable to give an expected protective effect.

The relationship between the ratio of n-3/n-6 PUFAs and lower breast cancer risk can be explained by the function of omega-3 PUFAs as an anti-cancer. $\alpha$-Linolenic acid (ALA, 18: $3 \mathrm{n} 3$ ) is the most common form of omega-3 PUFAs. The metabolism of ALA produces two important

Table 2. Association of Dietary Intake Ratio of n-3/n-6 Polyunsaturated Fatty Acids with Breast Cancer Risk in Western and Asian Countries

\begin{tabular}{lcccccc}
\hline Countries & \multirow{2}{*}{ Number of studies } & Pooled RR (95\% CI) & \multicolumn{2}{c}{ Heterogeneity } & \multicolumn{2}{c}{ Bias publication } \\
& & & $\mathrm{I}^{2}$ & $p$ & Begg's test & Egger's test \\
\hline Western & 10 & $0.98(0.91-1.06)$ & $57.00 \%$ & 0.013 & 0.009 & $<0.001$ \\
Asian & 3 & $1.18(0.94-1.47)$ & $47.20 \%$ & 0.151 & 0.117 & 0.24 \\
\hline
\end{tabular}

RR, Relative Risk; CI, Confidence Interval; $I^{2}$, Significant Heterogenity $>50 \% ; \mathrm{p}<0.05$ considered statistically significant 


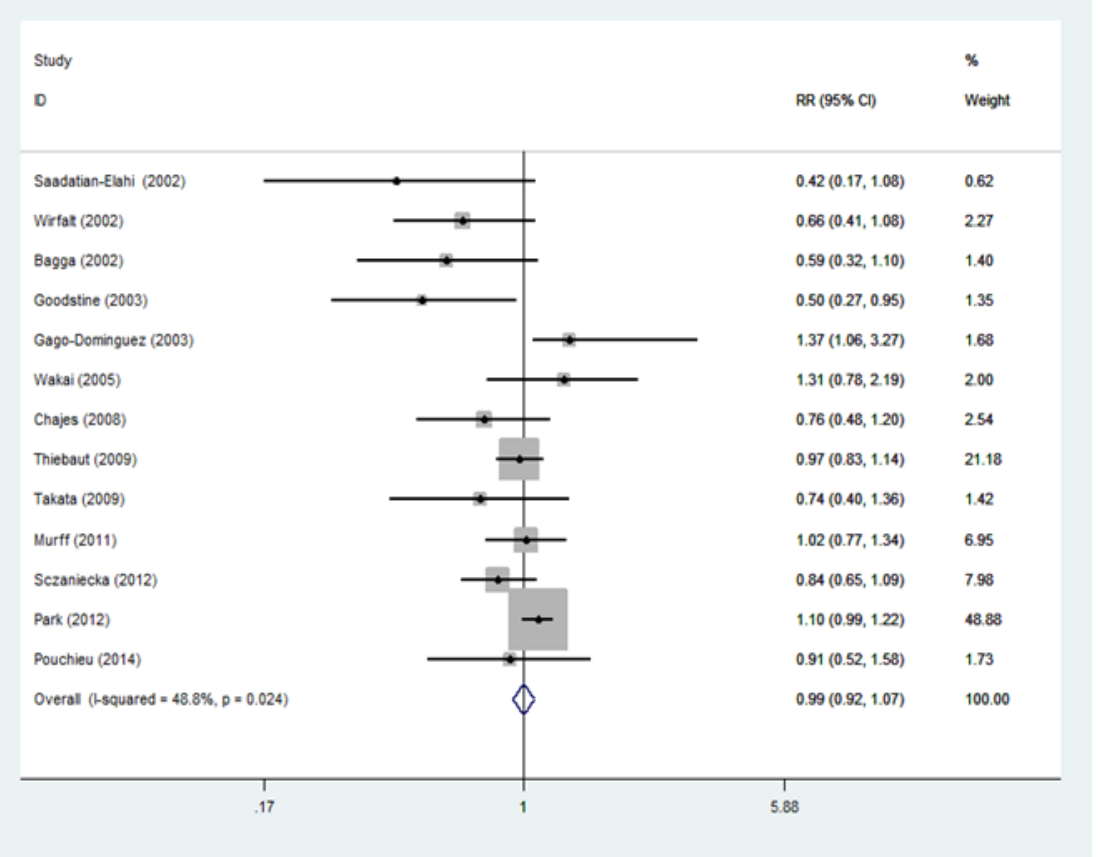

Figure 2. Forest Plots on Dietary Intake Ratio of n-3/n-6 Polyunsaturated Fatty Acids and Breast Cancer Risk in Western and Asian Countries

omega-3 PUFAs: eicosapentaenoic acid (EPA, 20: 5n3) and docosahexaenoic acid (DHA, 22: 6n3). EPA and DHA are found in seafood and fish oil. Fish products and fish oil are the main sources of omega-3 for diet (Willet, 2000; MacLennan and Ma, 2010).

The omega 6 fat diet affects the synthesis of eicosanoids, which will affect carcinogenesis through inflammatory and immunity responses, which then will affect the process of cell angiogenesis and mitosis. Meanwhile, omega-3-derived fatty acids are also able to give anti-inflammatory effects. Arachidonic acid, which is in a class of omega- 6 from long chain unsaturated fatty acids produced in the body, is able to stimulate tumor growth and metastasis. In contrast, eicosapentaenoic acid (EPA) and docosahexaenoic acid (DHA), from omega-3 fatty acids, inhibit growth and proliferation, and stimulate apoptosis in breast cancer cells, by suppressing and inhibiting the production of arachidonic acid (Nindrea et al., 2019; Shapira, 2017).

Angiogenesis, the key process for tumor growth and metastasis, occurs through the synthesis of omega- 6 fatty acids. However, diets high in omega-3 could provide

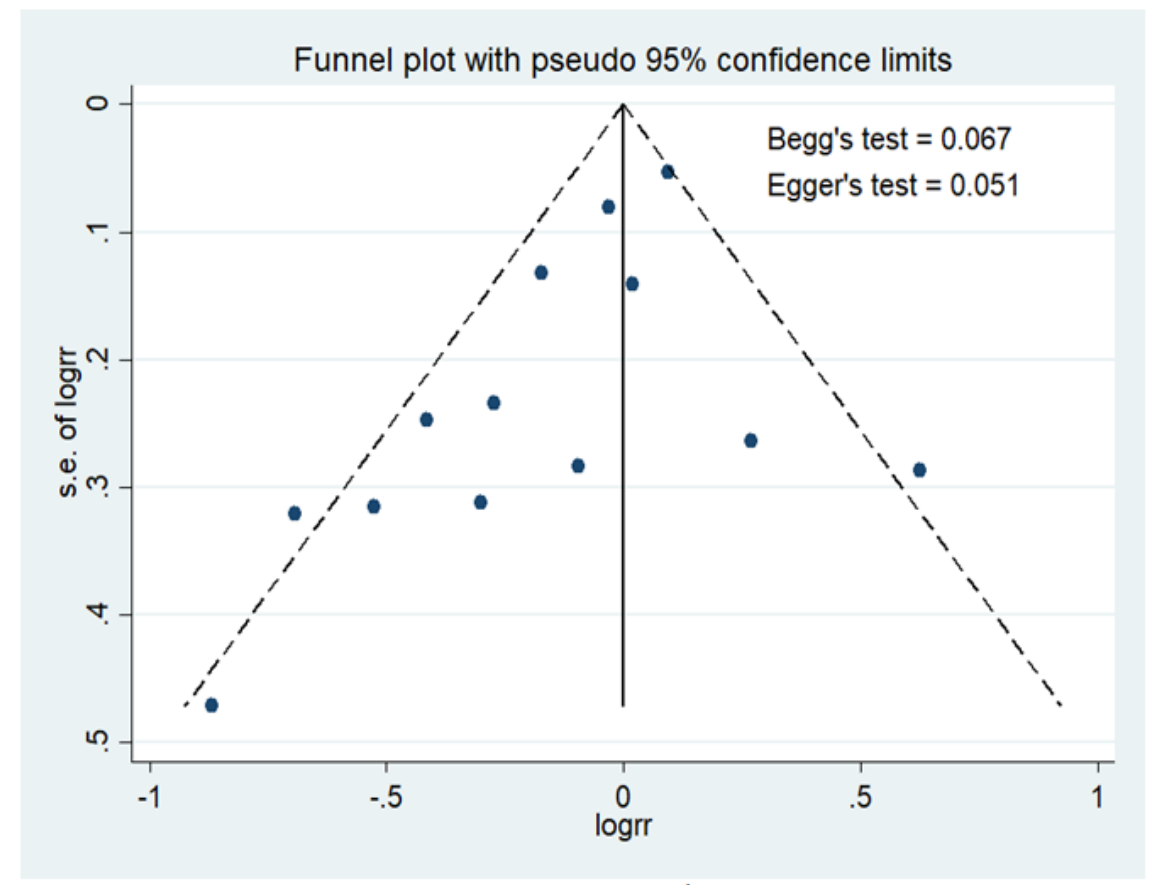

Figure 3. Funnel Plots on Dietary Intake Ratio of n-3/n-6 Polyunsaturated Fatty Acids with Breast Cancer Risk in Western and Asian Countries 
antiangiogenic properties by suppressing stimulation of omega-6 PUFA from breast cancer growth, metastasis and tumor vascularization. These fatty acids also affect the progression of cancer by affecting estrogen synthesis. Prostaglandin E2, and arachidonic fatty acids increase estrogen synthesis by increasing the expression and activity of aromatase enzymes. Different from EPA and oleic acid, as they decrease the production of prostaglandin E2, which will result in decreased estrogen production and consequently decreased estrogen effect (Makrem et al., 2013; Laviano et al., 2013).

The results of the study show that omega- 6 PUFA accelerates tumorigenesis, which is contradictory with omega-3 PUFA which has an anti-cancer effect. The western diet has a characteristic of lower omega-3 PUFA and higher omega-6 PUFA compared to traditional Asian diets. A study showed that Asian women who usually have high fish consumption as well as low incidence of breast cancer, showed an increased incidence of breast cancer after one generation of migration to Western countries. Intake of omega-3 and omega-6 PUFAs is usually estimated to be equal and balanced. But lately the western diet is very high in omega-6 PUFAs so the results showed an increase in the ratio of n-6/n-3 PUFAs (Gago-Dominguez et al., 2003; Wakai et al., 2005; Murff et al., 2011).

There were several limitations in this meta-analysis review. First, three studies seemed potentially eligible to be included in this meta-analysis but the full-texts were not accessible. Second, nine studies seemed potentially eligible to be included in this meta-analysis but they had insufficient data and irrelevant risk factors for data calculation. The second issue may increase the possibility of selection bias. Third, the number of cases sample in one study was relatively small as it can reduce the statistical strength (Bagga et al., 2002).

This study suggests for breast cancer prevention is by increasing dietary intake ratio of n-3/n-6 PUFAs. Based on dietary intake ratio of n-3/n-6 PUFAs, Asian population has more significant protection from breast cancer rather than Western population. The results of this study recommend the need to maintain dietary intake. Higher dietary intake ratio of n-3/n-6 PUFAs could possibly effective for breast cancer prevention. This study also suggests the need for education and counseling about eating habits and the importance of consuming foods with high fish compotition.

\section{Conflict of interest}

The authors declare no conflict of interest.

\section{Acknowledgements}

The authors extend their thanks to Dana Masyarakat (DAMAS) grant of Faculty of Medicine, Public Health and Nursing Universitas Gadjah Mada 2018 for help this research and Riyani Betri Novialita for collecting data.

\section{References}

Bagga D, Anders KH, Wang HJ, Glaspy JA (2002). Long-chain n-3-to-n-6 polyunsaturated fatty acid ratios in breast adipose tissue from women with and without breast cancer. Nutr Cancer, 42,180-5

Chajes V, Thiebaut AC, Rotival M, et al (2008). Association between serum trans-monounsaturated fatty acids and breast cancer risk in the E3N-EPIC Study. Am J Epidemiol, 167, 1312-20.

Engeset D, Alsaker E, Lund E, et al (2006). Fish consumption and breast cancer risk. The European Prospective Investigation into Cancer and Nutrition (EPIC). Int J Cancer, 119, 175-82.

Fabian CJ, Kimler BF, Hursting SD (2015). Omega-3 fatty acids for breast cancer prevention and survivorship. Breast Cancer Res, 17, 62.

Gago-Dominguez M, Yuan JM, Sun CL, et al (2003). Opposing effects of dietary n-3 and n-6 fatty acids on mammary carcinogenesis: The Singapore Chinese Health Study. $\mathrm{Br} J$ Cancer, 89, 1686-92.

Goodstine SL, Zheng T, Holford TR, et al (2003). Dietary (n-3)/ (n-6) fatty acid ratio: possible relationship to premenopausal but not postmenopausal breast cancer risk in U.S. women. J Nutr, 133, 1409-14.

Laviano A, Rianda S, Molfino A, Rossi-Fanelli F (2013). Omega-3 fatty acids in cancer. Curr Opin Clin Nutr Metab Care, 16, 156-61.

Liberati A, Altman DG, Tetzlaff J, et al (2009). The PRISMA statement for reporting systematic reviews and meta-analyses of studies that evaluate healthcare interventions: explanation and elaboration. BMJ, 339, b2700.

Liu J, Ma DW (2014). The role of n-3 polyunsaturated fatty acids in the prevention and treatment of breast cancer. Nutrients, 6, 5184-223.

MacLennan M, Ma DW (2010). Role of dietary fatty acids in mammary gland development and breast cancer. Breast Cancer Res, 12, 211.

Makarem N, Chandran U, Bandera EV, Parekh N (2013). Dietary fat in breast cancer survival. Annu Rev Nutr, 33, 319-48.

Murff HJ, Shu XO, Li H, et al (2011). Dietary Polyunsaturated fatty acids and breast cancer risk in Chinese women: a prospective cohort study. Int J Cancer, 128, 1434-41.

Nindrea RD, Aryandono T, Lazuardi L (2017). Breast cancer risk from modifiable and non-modifiable risk factors among women in Southeast Asia: a meta-analysis. Asian Pac J Cancer Prev, 18, 3201-6.

Nindrea RD, Aryandono T, Lazuardi L, Dwiprahasto I (2018). Diagnostic accuracy of different machine learning algorithms for breast cancer risk calculation: a meta-analysis. Asian Pac J Cancer Prev, 19, 1747-52.

Nindrea RD, Aryandono T, Lazuardi L, Dwiprahasto I (2019). Protective effect of omega-3 fatty acids in fish consumption against breast cancer in Asian patients: a meta-analysis. Asian Pac J Cancer Prev, 20, 327-32.

Nindrea RD, Aryandono T, Lazuardi L, Dwiprahasto I (2019). Family history of breast cancer and breast cancer risk between Malays ethnicity in Malaysia and Indonesia: A Meta-Analysis. Iran J Public Health, 42, 198-05.

Park SY, Kolonel LN, Henderson BE, Wilkens LR (2012). Dietary fat and breast cancer in postmenopausal women according to ethnicity and hormone receptor status: the multiethnic cohort study. Cancer Prev Res (Phila), 5, 216-28.

Pouchieu C, Chajes V, Laporte F, et al (2014). Prospective associations between plasma saturated, monounsaturated and polyunsaturated fatty acids and overall and breast cancer risk - modulation by antioxidants: a nested case-control study. PLoS One, 9, e90442.

Saadatian-Elahi M, Toniolo P, Ferrari P, et al (2002). Serum fatty acids and risk of breast cancer in a nested case-control study 
of the New York University Women's Health Study. Cancer Epidemiol Biomarkers Prev, 11, 1353-60.

Sczaniecka AK, Brasky TM, Lampe JW, et al (2012). Dietary intake of specific fatty acids and breast cancer risk among postmenopausal women in the VITAL cohort. Nutr Cancer, 64, 1131-42.

Shapira N (2017). The potential contribution of dietary factors to breast cancer prevention. Eur J Cancer Prev, 26, 385-95.

Takata Y, King IB, Neuhouser ML, et al (2009). Association of serum phospholipid fatty acids with breast cancer risk among postmenopausal cigarette smokers. Cancer Causes Control, 20, 497-504.

Terry PD, Rohan TE, Wolk A (2003). Intakes of fish and marine fatty acids and the risks of cancers of the breast and prostate and of other hormone-related cancers: a review of the epidemiologic evidence. Am J Clin Nutr, 77, 532-43.

Thiebaut AC, Chajes V, Gerber M, et al (2009). Dietary intakes of omega- 6 and omega-3 polyunsaturated fatty acids and the risk of breast cancer. Int $J$ Cancer, 124, 924-31.

Wakai K, Tamakoshi K, Date C, et al (2005). Dietary intakes of fat and fatty acids and risk of breast cancer: A prospective study in Japan. Cancer Sci, 96, 590-9.

Wells GA, Shea B, O'Connell D, et al (2009). The NewcastleOttawa Scale (NOS) for assessing the quality of nonrandomised studies in meta-analyses. [cited 2018 May 5]. Available from: http://www.ohri.ca/programs/clinical epidemiology/oxford.asp.

Willet WC (2000). Diet and cancer. Oncologist, 5, 393-04.

Wirfalt E, Mattison I, Gullberg B, et al (2002). Postmenopausal breast cancer is associated with high intakes of omega6 fatty acids (Sweden). Cancer Causes Control, 13, 883-93.

Yang B, Ren XL, Fu YQ, Gao JL, Li D (2014). Ratio of n-3/n-6 PUFAs and risk of breast cancer: a meta-analysis of 274135 adult females from 11 independent prospective studies. $B M C$ Cancer, 14, 105.

Zheng JS, Hu XJ, Zhao YM, et al (2013). Intake of fish and marine n-3 polyunsaturated fatty acids and risk of breast cancer: meta-analysis of data from 21 independent prospective cohort studies. $B M J, \mathbf{3 4 6}, \mathrm{f} 3706$.

\section{c) (7) (2)}

This work is licensed under a Creative Commons AttributionNon Commercial 4.0 International License. 